\title{
Characterization of Staphylococcus aureus strains from skin and soft tissue infection cases in Haripur and Abbottabad cities of Pakistan
}

\author{
Ayaz Saleem ${ }^{1}$, Neelum Ashfaq ${ }^{1}$, Mohammad Ejaz ${ }^{1,2}$, Bushra Fatima $^{1}$, Maria Gul ${ }^{1}$, Faisal Zaman ${ }^{3}$, Izhar ul Haq ${ }^{1}$, Bilal $^{2}$ \\ Ahmed ${ }^{2}$, Muhammad Ali Syed ${ }^{* 1}$ \\ ${ }^{1}$ Department of Microbiology, The University of Haripur, Haripur, 22650, Pakistan. \\ ${ }^{2}$ Department of Microbiology Quaid-i-Azam University, Islamabad,45320, Pakistan \\ ${ }^{3}$ Department of Medical Lab Technology, The University of Haripur, Haripur,22650, Pakistan \\ Corresponding Author's Email: mirwah2000@yahoo.com
}

\begin{abstract}
The current article reports the determination of the level of antibiotic resistance and detection of $p v l$ and mecA gene in Staphylococcus aureus strains isolated from skin and soft tissue infection cases at District Headquarters Hospital (DHQ) Haripur, Ayub Medical Complex, Abbottabad, Pakistan and Yahya Hospital, Haripur. A total of 405 samples were collected during the period from June 2018 and March 2019. Isolates were identified by using different microbiological, biochemical tests as well as cultural characteristics and polymerase chain reaction. The antibiotic sensitivity testing was carried out by using conventional disc diffusion assay, whereas the presence of Panton-Valentine Leukocidin $(P V L)$ and mecA genes in the isolated strains was determined using PCR. The results established that the 105 samples showed the presence of Staphylococcus aureus (S. aureus) (25.9\%), of them 84 (80\%) of the isolated strains have been identified as methicillin-resistant $S$. aureus (MRSA). A high level of resistance was obtained against cefazolin (91.43\%), augmentin (83.80\%), ceftriaxone (59.4\%), and lincomycin (57.10\%). No isolate was found to be resistant to vancomycin. The antibiotic-resistant gene mecA was found to be positive in all $100 \%(\mathrm{n}=80)$ samples with MRSA strains while $P V L$ gene was positive among 36\% ( $\mathrm{n}=38 \%$ ) of MRSA strains. The level of antibiotic resistance is higher than all previous studies from the region.
\end{abstract}

Keywords: Staphylococcus aureus, Antibiotic Resistance, MRSA, mecA, Panton-Valentine Leukocidin

\section{Highlights}

$>\quad$ Staphylococcus aureus strains, isolated from skin and soft tissues of the infected patients

$>\quad$ Detection of antibiotic resistance of gene mecA and gene encoding for virulence factor PVL form isolated strains

$>\quad$ Higher antibiotic resistance level than previous reports from the region

\section{Introduction}

Staphylococcus aureus is a notorious bacterial species causing a range of infections in the human body. Although, S. aureus is human commensal bacteria, it may cause many serious infections such as impetigo, furuncles, staphylococcal scalded skin syndrome (SSSS), pneumonia, otitis media, urinary tract infections as well as foodborne illnesses (Harris et al., 2002; Batabyal et al., 2012).

Skin and soft tissue infections (SSTIs) occur everywhere in the world and are among the most common types of infections. SSTIs are characterized by inflammatory microbial invasion of the epidermis, dermis, and subcutaneous tissues (Esposito et al., 2017). Skin is the largest body part that is exposed to the outside environment, with the underlying soft tissue, which includes the fat layers, fascia, and muscle, representing the majority of the tissues in the body. It acts as a tough, flexible, structural barrier to invasion. Therefore, the bacterial species invading skin must be equipped with several invasive enzymes and toxins as well as defense systems to overcome host factors (Perl and Roy, 1995). S. aureus is one of the major causes of skin wound infections, and is found to be associated with both community and hospital-acquired infections. Surgery and prolonged hospital stay predispose to postoperative S. aureus SSTIs (Perl \& Roy, 1995). Similarly, community-acquired SSTIs caused by $S$. aureus is also ubiquitous, which may become complicated (Stryjewski \& Chambers, 2008).

Antibiotics have been used for the treatment of $S$. aureus infections since their introduction in the 1940s. Previous studies have demonstrated that postsurgical wound infections have been difficult to be treated with conventional antibiotics (Idrees et al., 2009). Resistant strains emerged soon in the 1950s and their spread continues persistent to date. For instance, resistance to beta-lactamase resistant antibiotics e.g. methicillin appeared in 1960 while the resistant strains have spread to almost all over the world (Nwankwo et al., 2010). 
S. aureus once considered susceptible to cephalosporin and vancomycin, is now found to be resistant in recent studies. Both antibiotics failed to be a promising drug for treating S. aureus infections due to high level of resistance. Inappropriate use of antibiotics and time duration of dosage results in failure to treat the infection as well as give a shield to bacteria to develop resistance against that medicine (Coello et al., 1997, Hussain et al., 2005).

The prevalence of SSTIs caused by multiple resistant strains usually increases due to the extensive use of antibiotics. It was thought that the pathogens associated with community-acquired infections show resistance to fewer antibiotics, and may be susceptible to non- $\beta$-lactam antibiotics while the pathogens associated with hospital-acquired infection may be resistant to many of the antibiotics. However, the situation is worsening, since MRSA as well as resistant strains to all other antibiotics are equally being disseminated (Boucher and Corey, 2008, Crawford et al., 2006).

The $S$. aureus resistance against methicillin and other beta-lactam antibiotics is due to a gene known as mecA gene. $M e c A$ gene is about $2.1-\mathrm{kb}$ and located on SCC mec mobile genetic element. There are six different types of SCC mec elements isolated from $S$. aureus. From these types, type I, IV, and type V show resistance only against beta-lactam antibiotics while the presence of type II and III elements have been associated with resistance against multiple antibiotics including penicillin and kanamycin (Deurenberg and Stobberingh, 2009).

Some $S$. aureus strains produce a toxin called Panton-Valentine Leukocidin (PVL), which is responsible for tissue necrosis. PVL, encoded by the pvl gene, is an exotoxin that is expressed by only 2-3\% of wild types of S. aureus (Gillet et al., 2002). First CA-MRSA containing Panton-Valentine Leukocidin toxin was identified in the 1990s. Bacteriophage carries $p v l$ locus, which is present in only a very small number of $S$. aureus isolated from France while this PVL locus is linked with necrotic pneumonia as well as many skin infections (Gillet et al., 2002, Shore et al., 2014). Patients infected with PVL positive $S$. aureus strains show more hospitalization time duration than patients infected with PVL negative strains. Pro-phage transfer from other strains through horizontal gene transfer increases bacterial virulence (Herold et al., 1998, Hassan et al., 2008).

Molecular characterization of the isolated $S$. aureus strains helps its identification, source tracking, and comparison of the isolated bacterial strains with the ones in other areas. Staphylococcal protein A (Spa) typing, staphylococcal cassette chromosome (SSCmec) typing as well as detection of methicillin resistance ( $m e c A)$, and Panton-Valentine Leukocidin ( $p v l$ ) gene typing are some of the commonly used and economical methods of molecular characterization of $S$. aureus strains (Szabó, 2014).

Several public and private sector hospitals are located in Haripur and Abbottabad cities of Khyber Pakhtunkhwa and a high number of patients with skin and wound infections visited these hospitals and get treated. However, there is very limited data available on the contemporary antibiotic resistance profiles and molecular epidemiological characterization of the $S$. aureus strains. The purpose of the proposed research work was is to study antibiotic resistance patterns and to carry out molecular typing of the isolated strains from selected hospitals of Haripur and Abbottabad districts of Pakistan.

\section{Materials and Methods}

The cross-sectional study was carried out on randomly selected patients from different hospitals of Abbottabad and Haripur cities of Pakistan. Four hundred and five samples were analyzed during the period from June 2018 to March 2019 at Department of Microbiology, University of Haripur. Study participants were patients having skin and wound infections. Patient details such as type and site of infection, demographic details, treatment prescribed by the physician, and stay at the hospital were recorded. Informed consent was taken from all patients.

Bacterial swab samples were inoculated on a selective medium i.e. Mannitol Salt Agar (Oxoid, UK), followed by identification of Staphylococcus aureus using different biochemical tests and microscopy after their growth on selective media. Antibiotic sensitivity testing was performed using the Kirby-Bauer disc diffusion assay (Oxoid, UK). E-test strips (Biomerieux Company) were used for the determination of vancomycin resistance in the isolated S. aureus strains using manufacturer protocol. 


\subsection{DNA extraction and Polymerase Chain Reaction}

DNA was extracted using the phenol-chloroform method as described by Cheng and Jiang, (2006). mecA and $p v l$ genes were detected by PCR. Thermocycler of Eppendorf company and gel electrophoresis (Bio-Rad USA) was used for gene amplification and gel electrophoresis respectively. For the amplification of mecA gene mecA $\mathrm{F}-\left(5^{-}\right.$ GTAGAAATGACTGAACGTCCGATAA-3) and mecA R - (5-CCAATTCCACATTGTTTCGGTCTAA-3 ${ }^{-}$) primers were used, whereas pvl F- (5-ATCATTAGGTAAAATGTCTGGACATGATCCA-3) and pvl R- (5GCATCAAGTGTATTGGATAGCAAAAGC-3') were used for $p v l$ gene detection. The primer set nuc-1 (5'TCAGCAAATGCATCACAAACAG-3' and nuc-2(5'-GTAAATGCACTTGCTTCAGG-3') was used for PCR confirmation of $S$. aureus. All the selected primers were from a previous study (Poulsen et al., 2003).

\section{Results}

A total number of 405 samples were collected from Abbottabad and Haripur cities, 205 were from Abbottabad, and 200 from Haripur. Of all the collected samples, 105 shows positive results for $S$ aureus, 50 (52.4\%) of them were from Abbottabad, and $55(47.8 \%)$ from Haripur. Overall, the prevalence of $S$. aureus among skin and soft tissue infection cases in Haripur and Abbottabad cities found to be $27.5 \%$ and $24.5 \%$ respectively with no significant difference.

A high proportion of the isolated strains were found to be MRSA when tested against cefoxitin. The overall proportion of MRSA was found to be $80 \%(\mathrm{n}=84) ; 84.45 \%$ and $74 \%$ of the isolates from Haripur and Abbottabad districts respectively. Antibiotic sensitivity pattern of different antibiotics and their prevalence in Haripur and Abbottabad cities are shown in Figure 1.

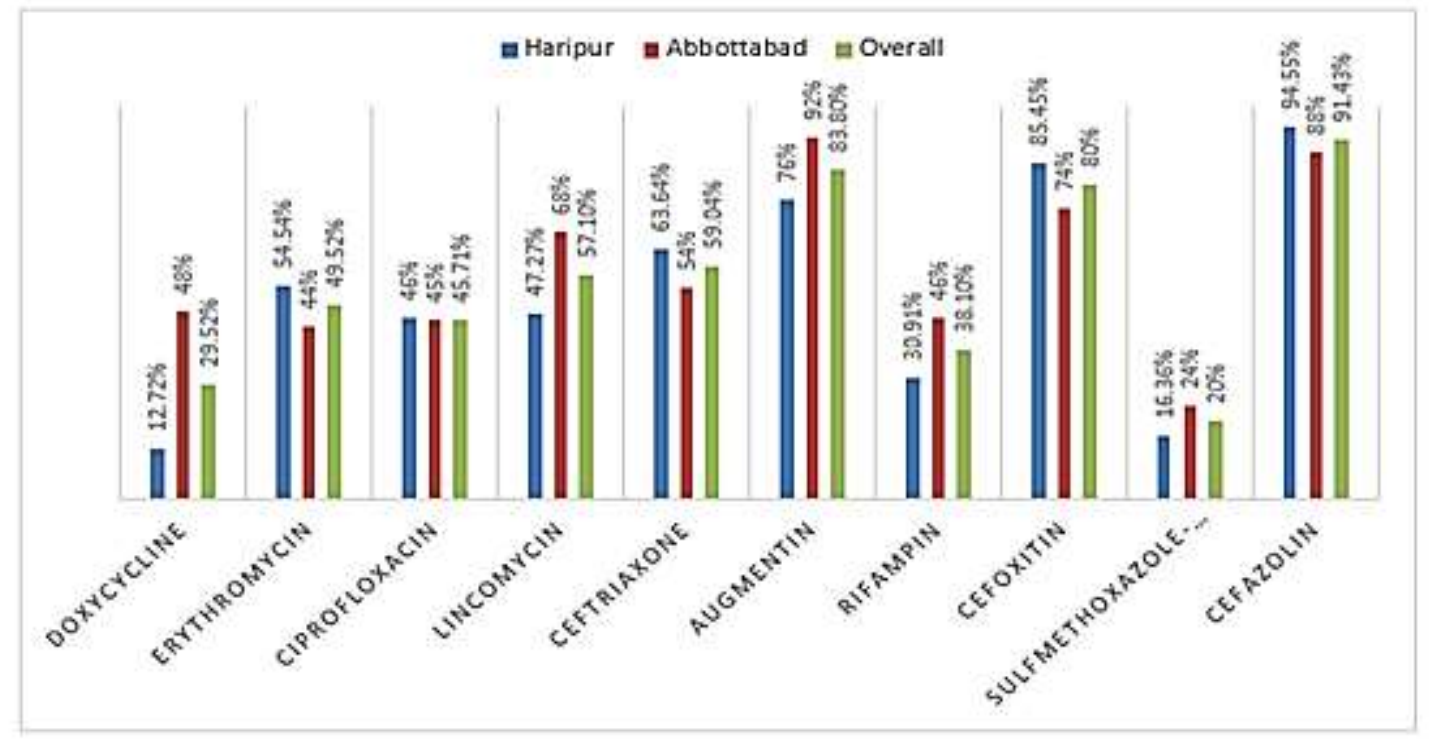

Fig.1. Antibiotic resistance profile of the isolated S. aureus strains from different patient's in Haripur and Abbottabad

The high level of resistance against different antibiotics was observed in the study. The overall resistance against cefazolin, augmentin, and cefoxitin were $91.43 \%, 83.80 \%$, and $80 \%$ respectively which was higher than previous data. While the resistance against doxycycline, ciprofloxacin, erythromycin, lincomycin, ceftriaxone, augmentin, rifampin, and sulfamethoxazole-trimethoprim shows $29.52 \%, 45.71 \%, 49.52 \%, 59.04 \%, 38.10 \%$ and $20 \%$ respectively.

The $n u c$ gene was used as a confirmatory test for S. aureus present among all $105(25.9 \%)$ isolates. $36 \%(\mathrm{n}=38)$ of all samples showed the presence of $p v l$ gene, among them $32.7 \%(n=18)$ isolates were from Haripur whereas $40 \%(n=20)$ were from Abbottabad had $p v l$ gene. mecA gene was present in all MRSA strains. Among 84 isolates of MRSA, 39.2\% ( $n=33$ ) were $m e c A$ positive in Haripur while $60 \%(\mathrm{n}=51)$ were positive in Abbottabad for mecA gene. Figure 2 shows the bands of $m e c A$ and $p v l$ genes after gel electrophoresis of amplified genes. 


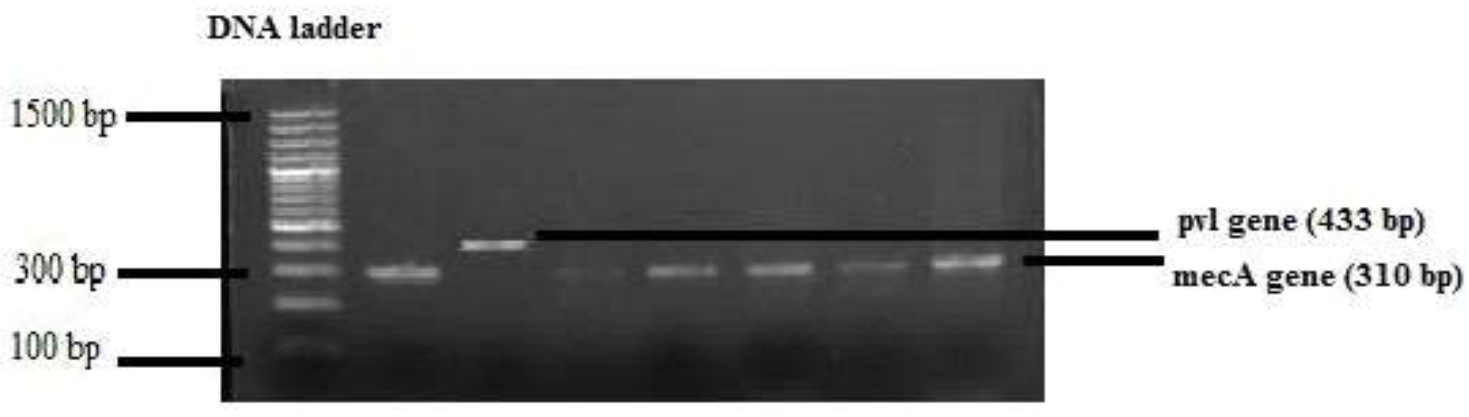

Fig. 2. Gel electrophoresis of mecA and pvl genes amplification process

Vancomycin is a glycopeptides antibiotic that is considered the drug of last resort in the case of complicated S. aureus infections. All isolates of our study were susceptible to E strip test of vancomycin.

\section{Discussion}

Antibiotic resistance has been witnessed as one of the most serious problems faced by health care professionals. Antibiotics were once considered magic bullets and hope for the human race that suffered a lot from infectious diseases in the course of history. It now has started failing due to bacterial resistance. Like many other developing countries, Pakistan is also a highly affected country, reporting a high level of microbial resistance against almost all medically important antibiotics to treat bacterial species. It is also evident from the results of the current study since majority of the isolated strains are resistant to conventionally used antibiotics against $S$. aureus. Eighty percent proportion of MRSA indicates an alarming situation and failure of the antibiotics once considered useful or drug of choice (Coello et al., 1997). The situation is even worse in the case of other antibiotics. A high level of resistance to all other antibiotics as well as the emergence of multidrug resistance highlights the seriousness of the issue as well as a challenge to the health care professionals facing nowadays. Compared with the previous studies conducted in Pakistan, this study reveals a higher prevalence of MRSA. A study conducted by Taj et al. (2010) in Karachi reported $38.6 \%$ strains resistant to methicillin, whereas the results of present study show $80 \%$ resistance, which is more than two-fold as compared to their report. A similar study conducted by Idrees et al. (2009) in Rawalpindi and Islamabad cities reported 53.3\% MRSA in 2010 (Khan et al., 2014).

Panton-Valentine Leukocidin is considered as one of the major virulence factors of $S$. aureus, especially those isolated from skin and soft tissue infections. However, recent studies suggest that $p v l$ positive strains are as common among $S$. aureus strains isolated from both communities acquired as well as hospital-acquired infections. In present study, the prevalence of $p v l$ gene found in both types of infections, which reveal this gene is to be found in both infection types (Shallcross et al., 2013).

\section{Conclusion}

In conclusion, the results of the present study are quite alarming since the majority of strains are showing a higher level of resistance to all tested antibiotics. This indicates the challenge faced by health professionals while treating skin and soft tissue infections in clinical practice. Furthermore, the proportion of MRSA strains is higher in our study as compared to previous reports from this region. None of the isolates identified as vancomycin resistant $S$. aureus, so vancomycin can still be considered as the drug of choice to treat complicated $S$. aureus infections.

\section{Conflict of Interest}

It is declaring that there is no conflict of interest in between Authors 


\section{Acknowledgment}

The authors highly acknowledge to Higher Education Commission (HEC) Pakistan for providing funds to support this research project under SRGP scheme.

\section{References}

1. Batabyal, Biswajit, Sukanta Chakraborty, and Shibendu Biswas. "Role of the oral micro flora in human population: A brief review." International Journal of Pharmacy \& Life Sciences 3.12 (2012).

2. Boucher, H. W., \& Corey, G. R. (2008). Epidemiology of methicillin-resistant Staphylococcus aureus. Clinical infectious diseases, 46(Supplement_5), S344-S349.

3. Cheng, H. R., \& Jiang, N. (2006). Extremely rapid extraction of DNA from bacteria and yeasts. Biotechnology letters, 28(1), 55-59.

4. Coello, R., Glynn, J. R., Gaspar, C., Picazo, J. J., \& Fereres, J. (1997). Risk factors for developing clinical infection with methicillin-resistant Staphylococcus aureus (MRSA) amongst hospital patients initially only colonized with MRSA. Journal of Hospital Infection, 37(1), 39-46.

5. Crawford, S. E., Boyle-Vavra, S., \& Daum, R. S. (2006). Community-Associated Methicillin-Resistant Staphylococcus aureus. Emerging infections 7, 153-179.

6. Deurenberg, R. H., \& Stobberingh, E. E. (2009). The molecular evolution of hospital-and community-associated methicillin-resistant Staphylococcus aureus. Current molecular medicine, 9(2), 100-115.

7. Esposito, S., De Simone, G., Gioia, R., Noviello, S., Pagliara, D., Campitiello, N., \& Ascione, T. (2017). Deep tissue biopsy vs. superficial swab culture, including microbial loading determination, in the microbiological assessment of Skin and Soft Tissue Infections (SSTIs). Journal of Chemotherapy, 29(3), 154-158.

8. Gillet, Y., Issartel, B., Vanhems, P., Fournet, J. C., Lina, G., Bes, M., \& Etienne, J. (2002). Association between Staphylococcus aureus strains carrying gene for Panton-Valentine leukocidin and highly lethal necrotising pneumonia in young immunocompetent patients. The Lancet, 359(9308), 753-759.

9. Harris, L. G., Foster, S. J., \& Richards, R. G. (2002). An introduction to Staphylococcus aureus, and techniques for identifying and quantifying S. aureus adhesins in relation to adhesion to biomaterials: review. Eur Cell Mater, 4(3), 100-20.

10. Hassan, K., Paturi, A., Hughes, C., \& Giles, S. (2008). The prevalence of methicillin resistant Staphylococus aureus in orthopaedics in a non-selective screening policy. The Surgeon, 6(4), 201-203.

11. Herold, B. C., Immergluck, L. C., Maranan, M. C., Lauderdale, D. S., Gaskin, R. E., Boyle-Vavra, S., ... \& Daum, R. S. (1998). Community-acquired methicillin-resistant Staphylococcus aureus in children with no identified predisposing risk. Jama, 279(8), 593-598.

12. Hussain, S., Shams, R., Ahmad, K., Perveen, R., \& Riaz, B. (2005). Prevalence of methicillin resistant Staphylococcus aureus (MRSA) in surgical site infections in a tertiary care hospital. Journal Pathol, 3(2), 81-85.

13. Idrees, F., Jabeen, K., Khan, M. S., \& Zafar, A. (2009). Antimicrobial resistance profile of methicillin resistant staphylococcal aureus from skin and soft tissue isolates. Journal of the Pakistan Medical Association, 59(5), 266.

14. Khan, S., Rasheed, F., \& Zahra, R. (2014). Genetic Polymorphism of agr Locus and Antibiotic Resistance of Staphylococcus aureus at two hospitals in Pakistan. Pakistan journal of medical sciences, 30(1), 172.

15. Nwankwo, B. O. K., Abdulhadi, S., Magagi, A., \& Ihesiulor, G. (2010). Methicillin resistant S. aureus (MRSA) and their antibiotic sensitivity pattern in Kano, Nigeria. African Journal of clinical and experimental microbiology, 11(1).

16. Perl, T. M., \& Roy, M. C. (1995). Postoperative wound infections: risk factors and role of Staphylococcus aureus nasal carriage. Journal of chemotherapy (Florence, Italy), 7, 29-35.

17. Poulsen, A. B., Skov, R., \& Pallesen, L. V. (2003). Detection of methicillin resistance in coagulase-negative staphylococci and in staphylococci directly from simulated blood cultures using the EVIGENE MRSA Detection Kit. Journal of Antimicrobial Chemotherapy, 51(2), 419-421.

18. Shabir, S., Hardy, K. J., Abbasi, W. S., McMurray, C. L., Malik, S. A., Wattal, C., \& Hawkey, P. M. (2010). Epidemiological typing of meticillin-resistant Staphylococcus aureus isolates from Pakistan and India. Journal of medical microbiology, 59(3), 330-337. 
19. Shallcross, L. J., Fragaszy, E., Johnson, A. M., \& Hayward, A. C. (2013). The role of the Panton-Valentine leucocidin toxin in staphylococcal disease: a systematic review and meta-analysis. The Lancet infectious diseases, 13(1), 43-54.

20. Shore, A. C., Tecklenborg, S. C., Brennan, G. I., Ehricht, R., Monecke, S., \& Coleman, D. C. (2014). PantonValentine leukocidin-positive Staphylococcus aureus in Ireland from 2002 to 2011: 21 clones, frequent importation of clones, temporal shifts of predominant methicillin-resistant S. aureus clones, and increasing multiresistance. Journal of clinical microbiology, 52(3), 859-870.

21. Stryjewski, M. E., \& Chambers, H. F. (2008). Skin and soft-tissue infections caused by community-acquired methicillin-resistant Staphylococcus aureus. Clinical Infectious Diseases, 46(Supplement_5), S368-S377.

22. Szabó, J. (2014). Molecular methods in epidemiology of Methicillin resistant Staphylococcus aureus (MRSA): advantages, disadvantages of different techniques. Journal of Medical Microbiology \& Diagnosis, 3(3), 1.

23. Taj, Y., Abdullah, F. E., \& Kazmi, S. U. (2010). Current pattern of antibiotic resistance in Staphylococcus aureus clinical isolates and the emergence of vancomycin resistance. J Coll Physicians Surg Pak, 20(11), 728-32. 